\title{
Papel de la microbiota intestinal en el desarrollo de la enfermedad de Alzheimer
}

\author{
Irene Fernández Martín (ifernmo2@estudiantes.unileon.es)
}

\begin{abstract}
Resumen
La enfermedad de Alzheimer (EA) es una enfermedad de carácter neurodegenerativo y la principal causa de demencia en el mundo. Cada vez más hipótesis apuntan a que es una enfermedad de origen intestinal: su patogénesis se ha relacionado con el eje microbiota-intestino-cerebro, y se especula que el desequilibrio intestinal puede estar vinculado con el desarrollo de la EA. La disbiosis hace que ciertos productos microbianos (amiloides, lipopolisacáridos y neurotransmisores) puedan atravesar la barrera intestinal y la hematoencefálica (BHE) y llegar al sistema nervioso central (SNC) aumentando el riesgo de padecer EA. En definitiva, la salud del SNC depende en gran medida de la salud de la microbiota intestinal y por ello los posibles tratamientos que se investigan para la EA se centran principalmente en la modulación de la microbiota mediante el uso de probióticos, prebióticos, trasplante de microbiota fecal y ejercicio físico.
\end{abstract}

Palabras clave: disbiosis, eje microbiota-intestino-cerebro, enfermedad de Alzheimer, microbiota, neuroinflamación.

\section{La enfermedad de Alzheimer}

La enfermedad de Alzheimer (EA) es una enfermedad de carácter neurodegenerativo y la principal causa de demencia en el mundo según la Organización Mundial de la Salud. Se caracteriza por la pérdida de memoria progresiva y trastornos conductuales (World Health Organisation, 2017). Es una de las enfermedades más comunes en personas de edad avanzada, suele manifestarse a partir de los 65 años (Reitzy Mayeux, 2014).

El primero en caracterizar la enfermedad fue Alöis Alzheimer en 1907. Describió los síntomas de Auguste Deter, una mujer de 51 años afectada por demencia. Tras su muerte realizó un examen cerebral encontrando: placas seniles, ovillos neurofibrilares y presencia de proteína $\beta$-amiloide (Fig. 1). Actualmente estas son las características neuropatológicas de la EA(Bondi et al., 2017).

\section{Agentes causales de la enfermedad de Alzheimer}

Hasta ahora se daba por hecho que la edad avanzada, los antecedentes familiares y genes de susceptibilidad eran los principales factores de riesgo (Reitz et al., 2011; Silva et al., 2019), sin embargo, el rápido aumento de la EA en la población no se puede explicar solo atendiendo a la genética (Hu et al., 2016). Esto 

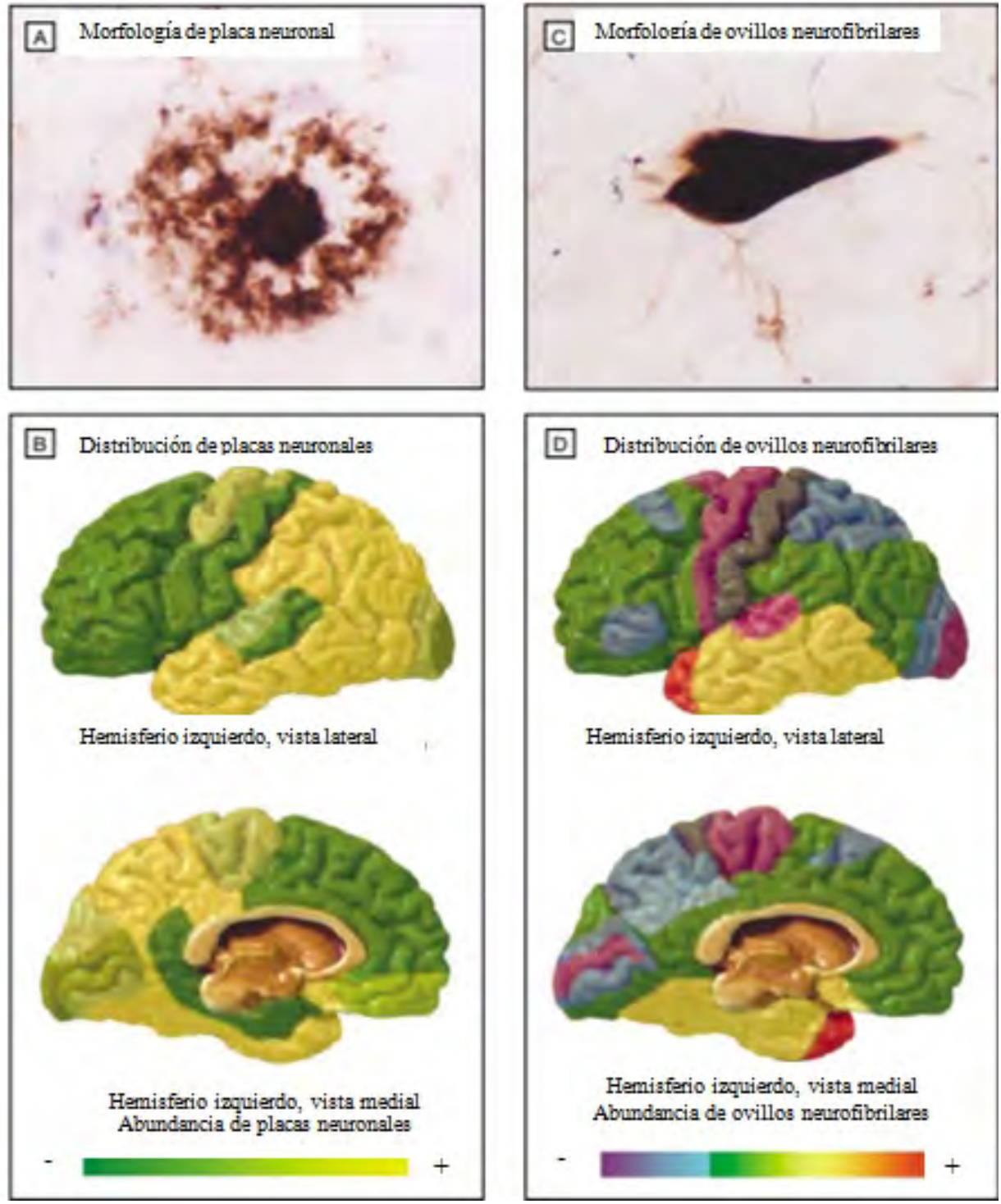

Figura 1. Placas neuronales y ovillos neurofibrilares: morfología (A y C) y distribución en el córtex cerebral (B y D) (Cummings et al., 2002)

nos lleva a pensar que los factores ambientales también juegan un papel crucial. Los principales factores de riesgo son enfermedades cerebrovasculares, hipertensión arterial, diabetes mellitus tipo 2 (DM2), elevado peso corporal, colesterol, tabaquismo, sedentarismo, dieta, escasa actividad intelectual entre otros (Fig. 2) (Lindsay et al., 2002; Campdelacreu, 2012; Hu et al., 2016; Bondi et al., 2017; Barragán et al., 2019; Serrano-Pozo y Growdon, 2019).

Sin embargo, en los últimos años se ha visto que otro factor importante que se podría asociar al desarrollo de EA es la alteración en la composición de la microbiota intestinal. Está relacionada con diversas enfermedades (obesidad, DM2, hipertensión, depresión, síndrome metabólico) y todas ellas son factores de riesgo de la EA(Hu et al., 2016). 


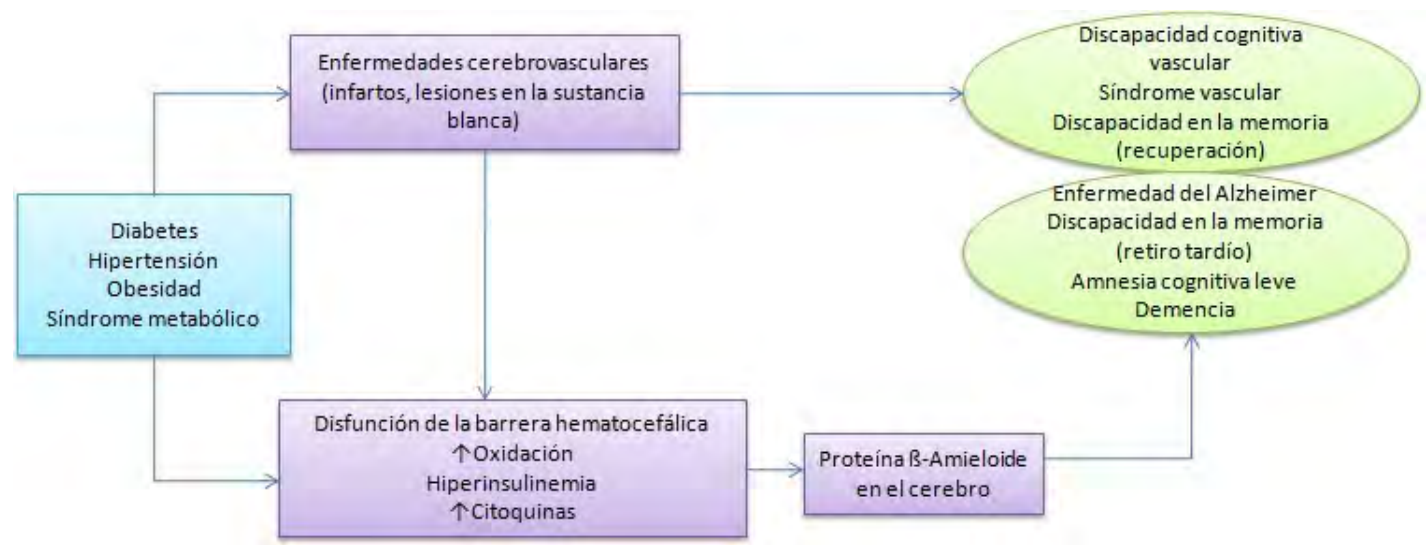

Figura 2. Principales mecanismos de interacción entre factores de riesgo vascular y disfunción cognitiva: enfermedades cerebrovasculares y acúmulos de proteína $\beta$-amiloide. Esquema adaptado de Reitzet al., 2011

\section{Microbiota intestinal}

Se entiende por microbiota intestinal la comunidad de microorganismos presentes de manera natural en el intestino, que conforman una relación simbiótica con el cuerpo humano, llevando a cabo funciones inmunes y metabólicas de manera conjunta (Hill et al., 2014; Rinninella et al., 2019). La microbiota intestinal está constituida en su mayoría por bacterias, pero también hay hongos, virus y protozoos. Los filos con más presencia son: Firmicutes (51\%) y Bacteroidetes (48\%) (Giau et al., 2018). No obstante, existen variaciones de la microbiota a diferentes niveles:

- Variabilidad interindividual: dependiendo del tipo de parto, si ha habido o no lactancia materna y cuánto ha durado esta, la dieta, el consumo de antibióticos, frecuencia con que se practica ejercicio... (Szablewski, 2017).

- Dentro del mismo individuo: la microbiota varía dependiendo de la zona intestinal que analicemos. El intestino delgado está menos colonizado, por su cercanía al estómago el pH es muy ácido y el tiempo de tránsito muy rápido. Mientras que en el intestino grueso el pH es más neutro y el tránsito más lento, alcanzándose la mayor abundancia de microorganismos en el colon (Passos y Morales-Filho, 2017; Rinninella et al., 2019).

- A lo largo de la vida: la colonización del tracto gastrointestinal comienza antes del nacimiento y posteriormente continua su diversificación hasta los 3 años (Passos y Morales-Filho, 2017; Giau et al., 2018). No obstante cuando ya está formada puede alterarse por consumo de antibióticos, estrés, las infecciones, el consumo de drogas, etc.(Hu et al., 2016). Finalmente a partir de los 70 años se ha observado una disminución en la diversidad 
de la microbiota, lo cual puede explicar la existencia de un sistema inmune más sensible y una absorción de nutrientes más pobre (Kim y Jazwinski, 2019).

Por todo ello no hay una microbiota "tipo" que sea la mejor, pero sí que debe existir un equilibrio entre bacterias beneficiosas (liberan sustancias antiinflamatorias) y perjudiciales (liberan sustancias proinflamatorias) (Rinninella et al., 2019).

\section{Eje microbiota-intestino-cerebro y relación con la enfermedad de} Alzheimer

El eje microbiota-intestino-cerebro tiene un carácter bidireccional y actúa a diferentes niveles: nervioso, endocrino, inmune y metabólico (Fig. 3). Cuando la composición de la microbiota intestinal se ve alterada (disbiosis) se produce una inflamación generalizada por el incremento de citocinas proinflamatorias. Esta inflamación es la que provoca que sea más permeable la barrera intestinal y se escapen productos metabólicos de las bacterias que pueden llegar al torrente sanguíneo, atravesar la barrera hematoencefálica (BHE) y desencadenar diferentes consecuencias en el sistema nervioso central (SNC). Estos acontecimientos parecen ser determinantes en el origen y evolución de la EA y otras enfermedades de carácter neurodegenerativo (Giau et al., 2018).

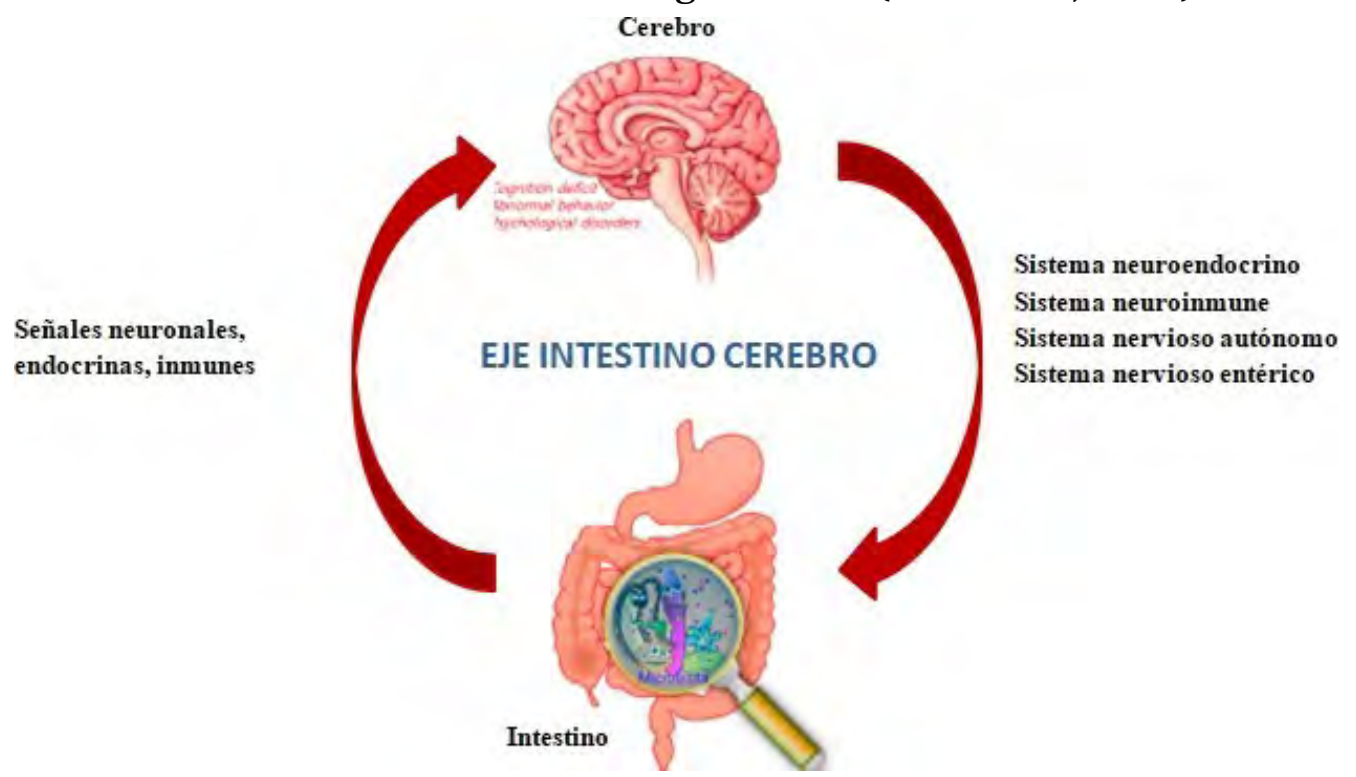

Figura 3. Regulación a tres niveles (hormonal, neuronal e inmunológico) de la señalización bidireccional del eje intestinocerebro. Imagen adaptada de Giau et al., 2018 
Como ya se ha mencionado, el problema surge cuando hay disbiosis intestinal. Unos microorganismos empiezan a ocupar el nicho de otros alterándose por completo la homeostasis de la microbiota (Shen, 2017; Szablewski, 2017). De manera simplificada, los principales metabolitos proinflamatorios liberados por las bacterias son amiloides y lipopolisacáridos (LPS). Su aumento provoca inflamación y permeabilización de la barrera intestinal. Si estos metabolitos consiguen pasar a la sangre se produce una inflamación sistémica, se permeabiliza la BHE y llegan hasta el SNC. En consecuencia, se origina neuroinflamación, característica de la EA (Kowalski y Mulak, 2019). Además, de manera análoga, infecciones bacterianas y víricas pueden ocasionar una situación de disbiosis que desencadena el mismo proceso (Szablewsky, 2017).

\section{Procesos patogénicos de la enfermedad de Alzheimer asociados a la microbiota}

- Los amiloides bacterianos son un producto bacteriano tóxico. Debido a su similitud el sistema inmune no discrimina entre los amiloides bacterianos y los amiloides del SNC (Kowalski y Mulak, 2019). Por ello se desencadenan graves respuestas de reacción autoinmune y en consecuencia una neuroinflamación vinculada con la EA (Jiang et al., 2017).

- Los LPS son el componente principal de la pared celular de bacterias Gram-negativas. En condiciones de permeabilidad son capaces de atravesar la barrera intestinal, la BHE y desencadenar reacciones de autoinmunidad en el SNC provocando una inflamación crónica característica de la EA (Szablewski, 2017).

Por ejemplo, se ha visto que la administración vía intraperitoneal de LPS en ratones provoca un aumento de agregaciones $\beta$-amiloides cerebrales propias de la patogenia de la EA. Estas se disponen en la zona del hipocampo que es la principal responsable de la memoria (Hu et al., 2016; Jiang et al., 2017).

- Inflamación y disfunción de la barrera intestinal. La barrera intestinal y la BHE se vuelven más permeables con la edad y es más fácil para los amiloides y el LPS atravesarla en edades avanzadas. Cuando estas sustancias son reconocidas por las células del sistema inmunitario innato se produce una liberación de citocinas proinflamatorias que provocan una inflamación crónica típica en la EA (Luca et al., 2019).

- La neuroinflamación es un síntoma definitorio de EA. En condiciones normales el aclaramiento de las sustancias tóxicas que llegan al cerebro es muy eficiente. Pero esta reacción inmune puede ser perjudicial si es continuada. Se ha visto que los pacientes de EA tienen la microglía y los astro- 
citos alterados (Calsolaro y Edison, 2016; Kowalski y Mulak, 2019).

- Procesos infecciosos y enfermedad de Alzhiemer. Se ha encontrado una relación entre la existencia de infecciones producidas por bacterias y virus y el desarrollo de EA. Helicobacter pylori es una bacteria del tracto gastrointestinal capaz de originar graves lesiones en la mucosa y el epitelio. Esta bacteria está fuertemente relacionada con la EA (Aguayo et al., 2018). Algo parecido ocurre con el virus del herpes simple tipo 1.

Concretamente un estudio mostró que los niveles plasmáticos de amiloides fueron significativamente más altos en pacientes de EA infectados con Borrelia burgdorferi, Chlamydia pneumoniae y $H$. pylori respecto a los individuos sanos (Angelucci et al., 2019).

\section{Terapias y fármacos para el tratamiento de la enfermedad de Alzheimer mediante la modulación de la microbiota}

Como hemos visto, numerosos indicios apuntan a que la EA es una enfermedad de carácter neurodegenerativo que se origina en el intestino a partir de una situación de disbiosis intestinal. Por ello, muchas líneas de investigación se centran principalmente en la modulación de la microbiota intestinal mediante la suplementación de la dieta con probióticos o prebióticos, e intervenciones en la microbiota que permitan restablecer su estado de homeostasis necesario como el trasplante fecal o la práctica de ejercicio físico (Jiang et al., 2017).

- Los probióticos son bacterias que en cantidades adecuadas aportan un beneficio a su hospedador (Sánchez et al., 2017). Se ha descrito que en ratones con EA tratados con probióticos se produce un aumento de hormonas beneficiosas para las funciones cognitivas, concretamente la grelina contrarresta el deterioro cognitivo de ratones con EA y la leptina protege del efecto tóxico de las agrupaciones $\beta$-amiloides (Distrutti et al., 2014).

- El término prebiótico hace referencia a las sustancias de la dieta que ocasionan cambios en la composición y/o actividad de la microbiota del tracto gastrointestinal favoreciendo a las bacterias beneficiosas (Liu et al., 2015). En un estudio llevado a cabo con ratones se usaron oligosacáridos extraídos de la planta Morinda officinalis como prebiótico. Los ratones mostraron un mejor rendimiento cognitivo y una disminución de placas de $\beta$-amiloide en el hipocampo que suele ser la zona más afectada en la EA (Chen et al., 2017).

- Trasplante fecal. El vínculo demostrado que existe en el eje microbiotaintestino- cerebro hace que cada vez surjan más terapias enfocadas hacia el trasplante de microbiota de un individuo sano a otro con disbiosis con la fi- 
nalidad de restablecer el equilibrio y revertir los daños en el SNC (Sun et al., 2019). Esto se lleva a cabo mediante trasplante fecal y los resultados de los estudios son prometedores.

- Ejercicio físico. Se ha demostrado que el ejercicio diario aumenta la diversidad de la microbiota (Karl et al., 2017). En consecuencia, las sustancias potencialmente peligrosas para el desarrollo de la EA que se originan en el intestino se quedan ahí y no llegan al SNC (Rinninella et al., 2019).

\section{Conclusiones}

- La causa de la enfermedad de Alzheimer permanece desconocida, pero la interacción entre genes de susceptibilidad y factores ambientales parece ser clave en su desarrollo.

- La principal hipótesis que describe el origen de la enfermedad de Alzheimer se asocia a la existencia de disbiosis, las funciones metabólicas e inmunes no se llevan a cabo de manera óptima y el eje microbiota-intestino-cerebro se ve alterado.

- La disbiosis es un factor común en pacientes con enfermedad de Alzheimer lo cual indica que la composición de la microbiota intestinal influye en la patogenia de la enfermedad.

- La homeostasis de la microbiota intestinal está claramente vinculada con la salud del sistema nervioso central y por ello las líneas de investigación se centran en terapias basadas en la modulación de la microbiota.

- Se ha demostrado que tanto los probióticos, como los prebióticos, el ejercicio físico y el trasplante de microbiota fecal son métodos efectivos contra la enfermedad de Alzheimer. No obstante, se necesitan muchos más estudios para verificar y esclarecer cómo la modulación de la microbiota produce cambios en el SNC y en qué medida son efectivos dichos tratamientos para los pacientes con enfermedad de Alzheimer.

En la Fig. 4 se representan, a modo de esquema, las conclusiones obtenidas que reflejan una visión general de los mecanismos patogénicos y las posibles aproximaciones terapéuticas basadas en la modulación de la microbiota. 


\section{ENFERMEDAD DE ALZHEIMER}

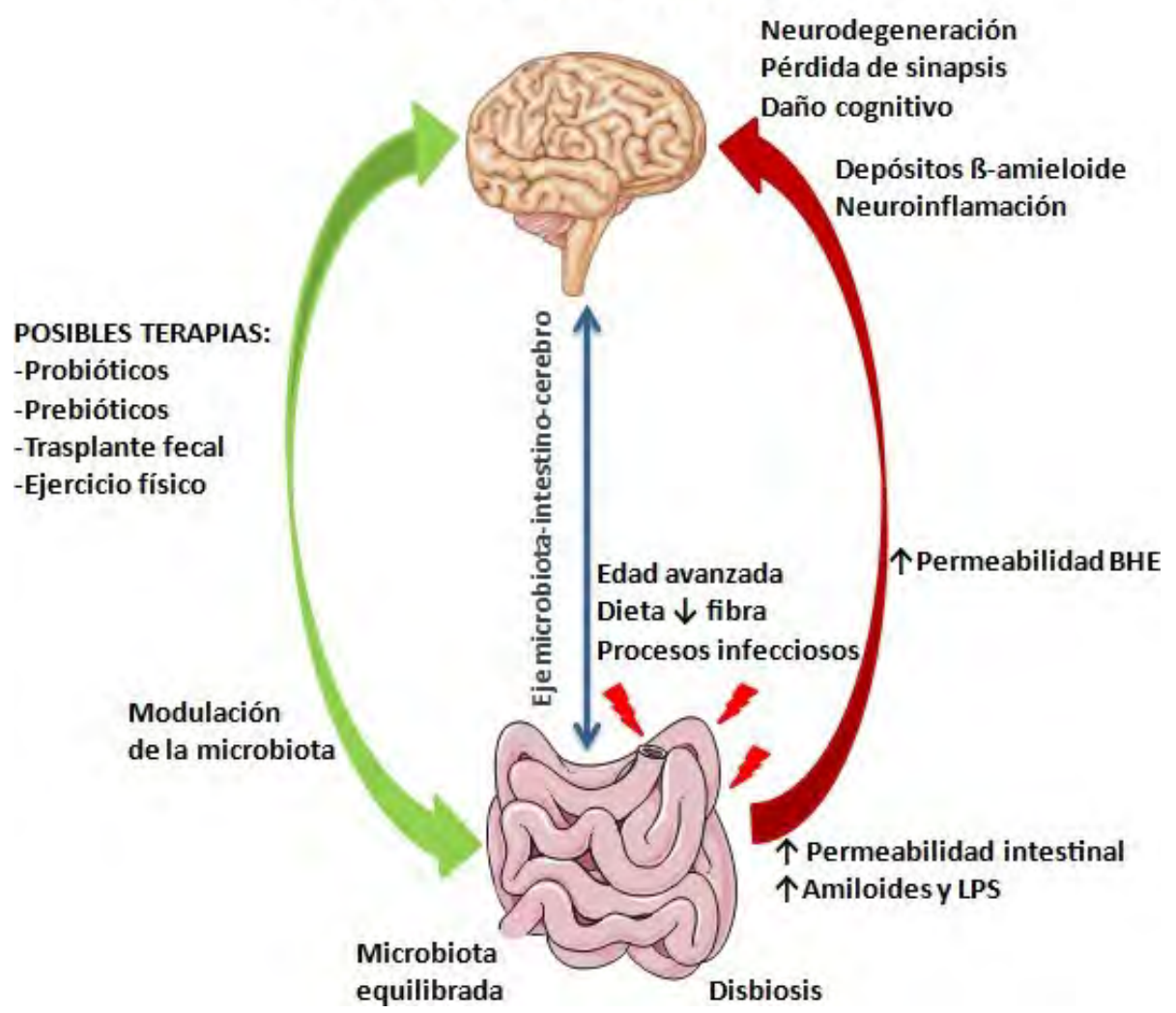

Figura 4. Representación esquemática de cómo la disbiosis afecta en la patogénesis de la enfermedad de Alzheimer mediante el eje microbiota-intestino-cerebro y las posibles terapias que revierten la situación para lograr una microbiota equilibrada

\section{Bibliografía}

Aguayo, S., Schuh, C. M. A. P., Vicente, B. y Aguayo, L. G. 2018. Association between Alzheimer's disease and oral and gut microbiota: are pore forming proteins the missing link?, Journal of Alzheimer's Disease, 65:29-46

Angelucci, F., Cechova, K., Amlerova, J. y Hort, J. 2019. Antibiotics, gut microbiota, and Alzheimer's disease, Journal of Neuroinflammation, 16:108

Barragán, D., García, M. A., Parra, A. y Tejero, J. 2019. Enfermedad de Alzheimer, Medicine, 12:4338-4346

Bondi, M. W., Edmonds, E. C., y Salmon, D. P. 2017. Alzheimer's disease: past, present and future, Journal of the International Neuropsychological Society, 23:818-831

Calsolaro, V. y Edison, P. 2016. Neuroinflammation in Alzheimer's disease: current evidence and future directions, Alzheimer's \& Dementia: the 
Journal of the Alzheimer's Association, 12:719-732

Campdelacreu, J. 2012. Enfermedad de Parkinson y enfermedad de Alzheimer: factores de riesgo ambientales, Neurología, 29:541-549

Chen, D., Yang, X., Yang, J., Lai, G., Yong, T., Tang, X., Shuai, O., Zhou, G., Xie, Y. y Wu, Q. 2017. Prebiotic effect of fructooligosaccharides from Morinda officinalis on Alzheimer's disease in rodent models by targeting the microbiota-gut-brain axis, Frontiersin Aging Neuroscience, 9(403)

Cummings, J. 2002. Alzheimer disease, Amerian Medical Association, 287:2335-2338

Distrutti, E., O’Reilly, J., McDonald, C., Cipriani, S., Renga, B., Lynch, M. M. y Fiorucci, S. 2014. Modulation of intestinal microbiota by the probiotic VSL\#3 resets brain gene expression and ameliorates the age-related deficit in LTP, Plos ONE, 9:1-11

Giau, V. V., Wu, S. Y., Jamerlan, A., An, S. S. A., Kim, S. Y. y Hulme, J. 2018. Gut microbiota and their neuroinflammatory implications in Alzheimer's disease, Nutrients, 10:1765

Hill, J. M., Bhattacharjee, S., Pogue, A. I. y Lukiw, J. 2014. The gastrointestinal tract microbiome and potential link to Alzheimer's disesase, Frontiers in Neurology, 5:1-4

Hu, X., Wang, T. y Jin, F. 2016. Alzheimer's disease and gut microbiota, Science China, 59:1006-1023

Jiang, C., Li, G., Huang, P., Liu, Z. y Zhao, B. 2017. The gut microbiota and Alzheimer's disease, Journal of Alzheimer's Disease, 58:1-15

Karl, J. P., Margolis, L. M., Madslien, E. H., Murphy, N. E., Castellani, J. W., Gundersen, Y., Hoke, A. V., Levangie, M. W., Kumar, R., Chakraborty, N., et al. 2017. Changes in intestinal microbiota composition and metabolism coincide with increased intestinal permeability in young adults under prolonged physiological stress, American Journal of Physiology. Gastrointestinal and Liver Physiology, 312:559-571

Kim, S. y Jazwinski, S. M. 2019. The gut microbiota and healthy aging, Gerontology, 64:513-520.

Kowalski, K. y Mulak, A. 2019. Brain-gut-microbiota axis in Alzheimer's disease, Journal of Neurogastroenterology and Motility, 25:48-60

Lindsay, J., Laurin, D., Verreault, R., Hébert, R., Helliwell, B., Hill, G. B. y McDowell, I. 2002. Risk factors for Alzheimer's disease: a prospective analysis from the canadian study of health and aging, American Journal of Epidemiology, 156:445-453

Liu, X., Cao, S. y Zhang, X. 2015 Modulation of gut microbiota-brain axis by probiotics, prebiotics, and diet, Journal of Agricultural and Food 
Chemistry, 63:7885-7895

Luca, M., Di Mauro, M., Di Mauro, M. y Luca, A. 2019. Gut microbiota in Alzheimer's disease, depression, and type 2 diabetes mellitus: the role of oxidative stress, Oxidative Medicine and Cellular Longevity, 2019, 1-10

Passos, M. C. F y Morales-Filho, J. P. 2017. Intestinal microbiota in digestive diseases, Arquivos de Gastreoenterologia, 54:255-262

Reitz, C., Brayne, C. y Mayeux, R. 2011. Epidemiology of Alzheimer's disease, Nature Reviews Neurology, 7:137-152

Reitz, C. y Mayeux, R. 2014. Alzheimer disease: epidemiology, diagnostic criteria, risk factors and biomarkers, Biochemical Pharmacology, 88:640-651

Rinninella, E., Raoul, P., Cintoni, M., Franceschi, F., Miggiano, G. A. D., Gasbarrini, A. y Mele, M. C. 2019. What is the healthy gut microbiota composition? A changing ecosystem across age, environment, diet and disease, Microorganisms, 7:1-22

Sánchez, B., Delgado, S., Blanco-Mínguez, A., Lourenço, A., Gueimonde, M. y Margolles, A. 2017. Probiotics, gut microbiota, and their influence on host health and disease, Molecular Nutrition \& Food Research, 61(1)

Serrano-Pozo, A. y Growdon, J. H. 2019. Is Alzheimer's disease risk modifiable?, Journal of Alzheimer's Disease, 67:795-819

Shen, T-C. D. 2017. Diet and gut microbiota in health and disease, Nestle Nutrition Institute Workshop Series, 88:117-126

Silva, M. V. F., Loures, C. de M. G., Alves, L. C. V., de Souza, L. C., Borges, K. B. G., y Carvalho, M. das G. 2019. Alzheimer's disease: risk factors and potentially protective measures, Journal of Biomedical Science, 26:1-11

Sun, J., Xu, J., Ling, Y., Wang, F., Gong, T., Yang, C., Ye, S., Ye, K., Wei, D., Song, Z., Chen, D. y Liu, J. 2019. Fecal microbiota transplantation alleviated Alzheimer's disease-like pathogenesis in APP/PS1 transgenic mice, Translational Psychiatry, 9:1-13

Szablewski, L. 2017. Human gut microbiota in health and Alzeimer's disease, Journal of Alzheimer's Disease, 62:549-560

World Health Organisation (2019) Dementia. Disponible en: https://www.who.int/news-room/fact-sheets/detail/dementia (Accedido: 26 defebrero de 2020) 